\title{
INTELLIGENT ELECTRIC POWER SYSTEMS WITH ACTIVE- ADAPTIVE ELECTRIC NETWORKS: CHALLENGES FOR SIMULATION TOOLS
}

\author{
Ruslan A. Ufa ${ }^{1 a}$, Venera A. Sulaymanova ${ }^{1}$ and Yuri S. Borovikov ${ }^{1}$ \\ ${ }^{1}$ National Research Tomsk Polytechnic University, 634050 Tomsk, Russia
}

\begin{abstract}
The motivation of the presented research is based on the needs for development of new methods and tools for adequate simulation of intelligent electric power systems with active-adaptive electric networks (IES) including Flexible Alternating Current Transmission System (FACTS) devices. The key requirements for the simulation were formed. The presented analysis of simulation results of IES confirms the need to use a hybrid modelling approach.
\end{abstract}

\section{Introduction}

The concept of intelligent electric power systems (IES) with active-adaptive electric networks is one of the modern trends of development and improvement of electric power systems (EPS) [1]. One of the main provisions of this concept is the introduction of Flexible Alternative Current Transmission System (FACTS) devices allowing transforming the largely passive electric network to activeadaptive and dramatically improving controllability in order to increase the capacity of power lines, improve the quality of electric energy, sustainability, ensuring efficient and reliable functioning of the EPS $[2,3]$.

However, the introduction of designated devices and technologies leads to significant changes and complication of the process in electric power equipment and the dynamic characteristics of the EPS as a whole. Accordingly, the working conditions of electric power equipment, relay protection system, technological and emergency control automatics, and, consequently, the setting of these systems is changed significantly and dynamically $[3,4]$.

Due to insufficient knowledge of above identified processes and operating conditions of the IES, comprehensive research, which assumes presence of sufficiently complete and reliable information about these processes, is very relevant. A solution of this task requires full-scale experiments in a real EPS, which cannot be conducted. Therefore, the adequate simulation remains the main tool for analyzing of IES $[5,6]$.

\section{Requirements to simulation tools}

The specifics of the functional requirements of FACTS devices should be considered to solve the problem of complete and adequate modeling of processes in the IES, in particular $[4,5,7]$ :

${ }^{\text {a }}$ Corresponding author : hecn@tpu.ru 
- the phase-phase operation of voltage-source converter (VSC) of FACTS devices, which eliminates the use of one-line diagram calculation of IES and method of symmetrical components;

- the continuous high-speed operation of FACTS devices in all possible normal, emergency and post-emergency operating conditions of IES, which eliminates the use of decomposition of processes, limitations on their duration (without separation of electromagnetic and electromechanical transient processes modeling in power equipment and IES as a whole) and static mathematical models not only FACTS devices, and other equipment of IES;

- the use of high-speed fully controlled power semiconductors, which determines, including realtime, prompt obtaining information about the processes in power equipment and EPS as a whole for a variety of operational tasks as well as for testing and development of algorithms of automatic control of FACTS devices.

The totality of the above mentioned specific properties of IES, taking into account the requirements for adequacy of the results of modeling, defines a set of features that should have the software and hardware simulation tools of IES with FACTS devices [4, 5, 7]:

- the models of IPS elements must be three-phase (or more) to account properly for all the unbalanced conditions;

- the simulation of IES must be with guaranteed acceptable accuracy in real time on the range of occurrence of a single continuous spectrum quasi-steady-state and transient processes in all possible normal, emergency and post-emergency operating conditions of IES.

A comparison of these requirements with the features and capabilities currently used by many software and software-hardware purely digital simulation shows their significant discrepancy on all these counts. This discrepancy is explained by using numerical integration methods, based on the notion of the nature of continuous processes in discrete form.

This feature of digital simulation tools leads to the need for various forms of decomposition simulated processes (separation of electromagnetic and electromechanical processes), find a compromise between the differential order of the simulated power system, the simulation time and the ability of applied the methods and means $[8,9]$.

In addition, independently of this methodological error of solution, corresponding to the method of numerical integration of differential equations, always remain unknown, the determination of the actual value of which is related to methods of sampling theory of differential equations to the category of fundamental problems. In part, these conclusions are confirmed by the results of the research and analysis of severe accidents with a digital model of the IES [10].

The results of the verification of digital models of Western United States based on Extended Transient Midterm Stability Program (ETMSP) software with data of the Wide Area Measurement Systems (WAMS) are shown in fig. 1.

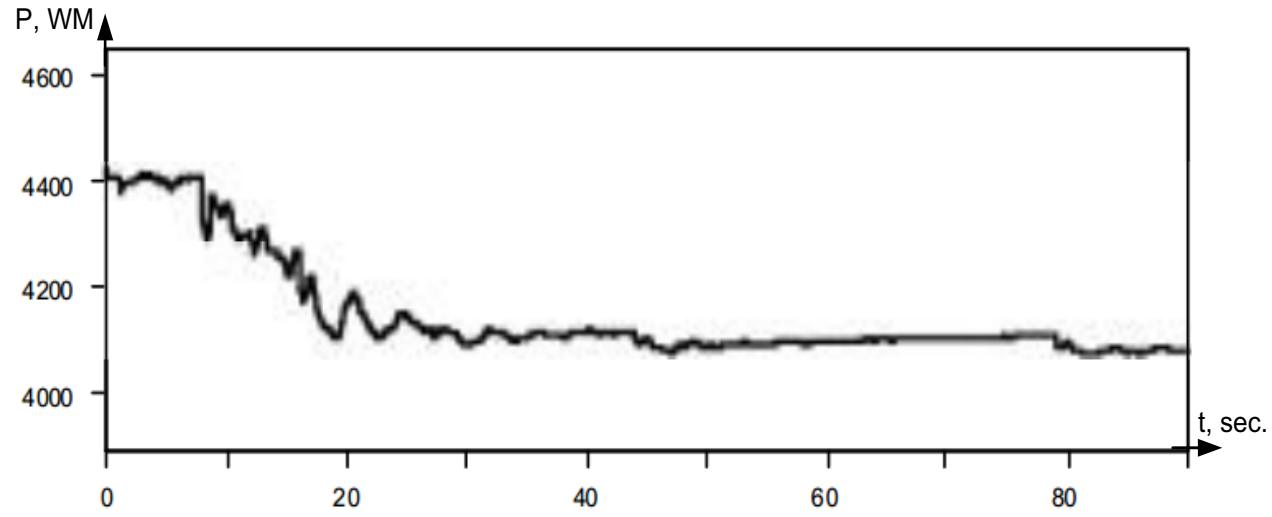




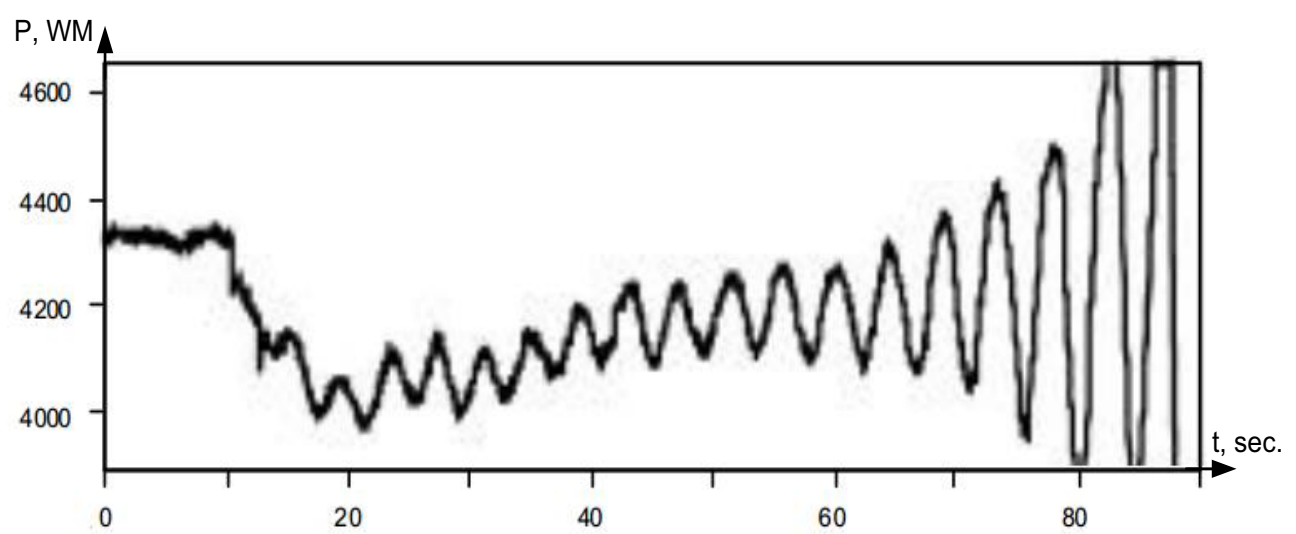

b)

Figure 1. Oscillogram of the power flow obtained by digital models (a) and WAMS (b).

According to fig. 1 obtained by digital models (fig. 1a) curves of regime parameters change are far from the actual recorded during the accident curves (fig. 1b), and the digital model does not reproduce actual accidents even qualitatively.

Indeed, in a real power system the development of synchronous oscillations occurs at a frequency of about $0.15-0.17 \mathrm{~Hz}$, which leads to disruption of stability. Digital model accurately shows only the initial phase of the disturbance on the range of the first 10-15 sec., and then calculated the electromechanical transient well damped, and the stability of parallel operation of the power system is maintained. Using simplified models of equipment and IES as a whole, which does not adequately reproduce the processes in IES as a whole, is one of the main reasons for inconsistencies of simulation results.

As a solution to the problem of obtaining sufficiently complete and reliable information about the processes in the IES a hybrid modeling approach based on using different modeling approaches is proposed $[11,12]$.

Hybrid Real-time Simulator of EPS (HRTSim), based on the use of three modeling approaches: analog, digital and physical, each of which achieves maximum efficiency in solving individual subtasks and whose architecture is discussed in more detail in $[12,13,14]$, is an example of a simulator developed on this hybrid modeling approach.

Experience of application of HRTSim for various research and operational problems [12, 13, 14] can reasonably argue about the effectiveness of solving the problem sufficiently complete and reliable real time simulation decomposition of processes on an unlimited time interval of equipment and IES as a whole.

The basic points of the HRTSim are:

- the power equipment of IES is described via complete systems of differential equations adequately representing the whole significant range of quasi-steady and transient processes in this equipment and forming comprehensive mathematical models of corresponding types of the simulated equipment;

- the methodologically accurate with guaranteed instrumental error solution of differential equation systems in real time and over an unlimited period of time are carried out by means of the continuous implicit integration method;

- all types of commutation of power equipment, including the power semiconductors, are carried out on a model physical level;

- the interconnection between a physical model and mathematical simulation levels is provided by means of appropriate voltage-current converters; 
- a mutual conversion of mathematical and model physical variables in conjunction with simulation on the physical model level of the commutation of power equipment provides the ability of unlimited scalability of the simulated IES;

- all informational and control functions, as well as modeling control and protection systems are implemented on a digital level using a digital-to-analog, analog-to-digital conversion and specialized local and server software.

\section{Conclusions}

Development of IES associate with the introduction of high-speed FACTS devices, having indicated in the article characteristic, eliminates the use of one-line diagram calculation of IES, method of symmetrical components, static mathematical models of FACTS devices and other equipment of IES, decomposition of processes (separation and division of electromagnetic and electromechanical transients), limiting playback interval.

- The carried out theoretical analysis of such reasons gives grounds for concluding that the fundamental insolvability of the problem within the existing unilateral purely numerical modeling approach of IES.

- The only way to solve this problem is complex approach assumes involves pooling and aggregation of different modeling approaches.

- Research and analysis developed in accordance with the mentioned comprehensive approach HRTSim can reasonably argue about the effectiveness of solving the problem sufficiently complete and reliable real time simulation decomposition of processes on an unlimited time interval of equipment and IES as a whole.

The work was supported by Project № 138_2014 "Hybrid simulation and control of smart grids".

\section{References}

1. P. Thepparat, D. Retzmann, E. Ogée, M. Wiesinger, Smart Transmission System by HVDC and FACTS (Grenoble, France, 2013)

2. Z. H. Liu, Y. Wang, J. M. Chen, Y. R. Guo, X. G. Wang, Z. Q. LI, D. X. Du, X. Li, Modeling and simulation research of large-scale AC/DC hybrid power grid based on ADPSS (Kowloon, Hong Kong, 2014)

3. M. Negnevitsky, N. Voropai, V. Kurbatsky, N. Tomin, D. Panasetsky, Development of an intelligent system for preventing large-scale emergencies in power systems (Vancouver, Canada, 2013)

4. Yu.S. Borovikov, A.S. Gusev, A.O. Sulaymanov, R.A. Ufa, Hybrid real-time simulator of power system for advanced simulation of the FACTS and HVDC system based on Voltage Source Converter (Shanghai, China, 2014)

5. D. Qi, Electra, 257, 4 (2011)

6. A. Tavlintsev, A. Pazderin, A. Suvorov, P. Chusovitin, O. Malozemova, Experimental investigation of static load characteristics (Xi'an, China, 2014)

7. XU Lin, Y. H. Tang, P. U. Wei, Y. Han, Journal of Energy in South Africa, 25, 112 (2014)

8. Y. Zhang, A.M. Gole, W. Wu, B. Zhang, H. Sun, IEEE Transaction on power system. 5, 357 (2013)

9. O. Nayak, S. Santoso, P. Buchanan, IEEE Computer Applications in Power, 15 (4), 37 (2002)

10. J. F. Hauer, M. J. Beshir, Dynamic Performance Validation in the Western Power System (Kananaskis, Alberta, 2000)

11. Chen, K.-J. Zhang, Y.-J. Xia, G. Hu, J. Electron. Sci. Technol, 11 (1), 66 (2013)

12. Yu. Borovikov, A. Prokhorov, M. Andreev, Application of Hybrid Real Time Simulator for solution of Smart Grid tasks on the example of Elgaugol energy cluster pilot project (Tomsk, Russia, 2012) 
13. N.Y. Ruban, Y. S. Borovikov, A. O. Sulaymanov, Simulation of transmission lines phasecomparison protection for optimization its settings, Strategic Technology (Cox's Bazar, Bangladesh, 2014)

14. Y.S. Borovikov, A.O. Sulaymanov. A.S. Gusev, M.V. Andreev, Simulation of automatic exciting regulators of synchronous generators in hybrid real-time power system (Shanghai, China, 2014) 